\title{
CAPTURA DE Rhynchophorus palmarum L. (COLEOPTERA: CURCULIONIDAE) EM AR- MADILHAS ISCADAS COM O FEROMÔNIO DE AGREGAÇÃO E COMPOSTOS VOLÁ- TEIS DE FRUTOS DO ABACAXI
}

\author{
ADRIANA GUIMARÃES DUARTE², IVANILDO SOARES DE LIMA³, DANIELA MARIA DO AMARAL FERRAZ \\ NAVARRO ${ }^{4}$, ANTÔNIO EUZÉBIO GOULART SANT'ANA ${ }^{5}$
}

\begin{abstract}
RESUMO - O objetivo deste trabalho foi determinar os índices de captura de Rhynchophorus palmarum em armadilhas iscadas com o feromônio de agregação, 6-metil-2(E)-hepten-4-ol (rincoforol), associado a toletes de cana-de-açúcar, a pedaços de frutos do abacaxi e a seis compostos voláteis isolados de frutos do abacaxi. Os compostos voláteis do abacaxi são caracterizados por uma mistura de ésteres metílicos e etílicos, sendo o octanoato de metila e o octanoato de etila os mais abundantes. As armadilhas iscadas com o rincoforol associado a toletes de cana-de-açúcar e as iscas com rincoforol associado a pedaços de abacaxi não apresentaram diferenças significativas no número de besouros capturados. No entanto, ambas apresentaram índices de captura superiores àquelas em que o rincoforol foi utilizado em associação com voláteis do abacaxi. Não se observaram efeitos significativos do local e época de captura, nem no número de machos e de fêmeas capturados.
\end{abstract}

Termos para indexação: Insecta, rincoforol, Ananas comosus, infoquímicos.

\section{TRAP CATCHES OF Rhynchophorus palmarum L. (COLEOPTERA: CURCULIONIDAE) BAITED WITH ITS AG- GREGATION PHEROMONE AND VOLATILE COMPOUNDS FROM PINEAPPLE}

\begin{abstract}
The aim of this work was to investigate the capture of Rhynchophorus palmarum in traps baited with its aggregation pheromone, 6methyl-2(E)-hepten-4-ol (rhynchophorol), in association with sugar cane, pieces of pineapple fruit, and six volatile compounds from pineapple. A mixture of methyl and ethyl esters, being methyl octanoate and ethyl octanoate the most abundant, characterizes the volatile compounds from pineapple fruits. Traps baited with rhynchophorol in association with sugar cane and those baited with rhynchophorol in association with pieces of pineapple, showed no significant differences in the number of trapped weevils. However, both traps caught significantly more weevils, than those baited with rhynchophorol in association with pineapple volatiles. There were no significant effects from place and time or in the number of male and female weevils trapped.
\end{abstract}

Index terms: Insecta, rhynchophorol, Ananas comosus, infochemicals.

\section{INTRODUÇÃO}

A cultura do coqueiro (Cocus nucifera L.) é de fundamental importância para a economia dos Estados do Nordeste do Brasil. Os insetos-praga limitam a sua exploração, porque são os principais responsáveis pela baixa produtividade da cultura. Os adultos e as larvas de Rhynchophorus palmarum (Coleoptera: Curculionidae) fazem galerias no meristema apical da planta, e os odores emanados dos tecidos atacados atraem outros indivíduos machos e fêmeas da espécie. Os machos de $R$. palmarum alimentam-se e liberam o feromônio de agregação, atraindo mais machos e fêmeas. Segundo Griffith (1968), uma população de 30 larvas é suficiente para matar uma planta adulta. Em plantas jovens, é possível que um número menor de larvas cause o mesmo efeito (Sánchez \& Cerda, 1993). Vários autores observaram larvas do gênero Rhynchophorus alimentando-se de uma grande variedade de plantas das famílias Arecaceae, Gramineae, Caricaceae, Bromeliaceae e Musaceae (Hagley, 1965; Wattanapongsiri, 1966). Em laboratório, larvas e adultos de R. palmarum são alimentados com frutos de abacaxi (Ananas comosus L.) e colmos de cana-de-açúcar, Saccharum officinarum L. (Giblin-Davis et al., 1989).

Pesquisas anteriores à identificação e síntese do feromônio de agregação de $R$. palmarum, 6-metil-2(E)-hepten-4-ol (rincoforol), já evidenciavam que alguns odores provenientes da fermentação de tecidos vegetais, tais como o etanol, atraem adultos de várias espécies do gênero Rhynchophorus (Hagley, 1965; Gunatilake \& Gunawardena, 1986). Armadilhas iscadas apenas com o feromônio de agregação, ou com partes de plantas hospedeiras, não são muito eficientes na captura dos besouros das palmeiras. Porém, quando combinados, o efeito sinérgico aumenta a atração em até 20 vezes (Oehlschlager et al., 1992; Gries et al., 1993; Perez et al., 1994; Oehlschlager et al., 1995). Devido a esse sinergismo, muitas substâncias voláteis, provenientes dos tecidos de palmeiras e de outras plantas hospedeiras, têm sido identificadas. Vários ésteres de palmeiras, como o acetato de etila, propionato de etila, butirato de etila e isobutirato de etila, testados em associação com o feromônio, aumentaram a atração de $R$. phoenicis, $R$. cruentatus, $R$. palmarum, $R$. bilineatus, $R$. ferrugineus e $R$. vulneratus (Gries et al., 1994). Esses mesmos autores também relataram que o propionato de etila foi o único éster testado capaz de aumentar a atração de $R$. phoenicis para as armadilhas iscadas com $( \pm)$-fenicol. $\mathrm{O}$ acetato de etila, propionato de etila, butirato de etila, isobutirato de etila e etanol, testados em várias dosagens, proporcionaram efeito sinérgico na atração de $R$. cruentatus para armadilhas iscadas com ( \pm )-cruentol (Giblin-Davis et al., 1994). Em proporções desconhecidas, o acetato de etila aumentou a atração de $R$. palmarum para armadilhas contendo ( \pm )-rincoforol (Jaffé et al., 1993).

Substâncias voláteis, provenientes da fermentação de toletes de cana-de-açúcar, acetato de etila, propionato de etila e butirato de etila, igualmente aumentaram a atratividade de Metamasius hemipterus para armadilhas contendo o feromônio metalure (Perez et al., 1995). Entretanto, nenhum dos ésteres testados foi tão eficiente na captura da broca-da-palmeira quanto pedaços de palmeiras ou de cana-de-açúcar em associação com o feromônio de agregação (Jaffé et al., 1993; GiblinDavis et al., 1994; Gries et al., 1994). Isso sugere que outros componentes cairomonais precisam ser adicionados e testados em diferentes proporções.

O objetivo deste trabalho foi determinar os índices de captura de Rhynchophorus palmarum em armadilhas iscadas com o feromônio de agregação, 6-metil-2(E)-hepten-4-ol (rincoforol), associado a toletes de cana-de-açúcar, a pedaços de frutos do abacaxi e a seis compostos voláteis isolados de frutos do abacaxi.

\footnotetext{
${ }^{1}$ Trabalho (128/2002). Recebido: 31/07/2002; Aceito para publicação: 20/03/2003

${ }^{2}$ Eng. Agrônoma, Ms, doutoranda, CPGQB - QUI/CCEN/UFAL. BR 104 Norte, Km 14, Tabuleiro do Martins, 57072-970, Maceió-AL. agd@fapeal.br.

${ }^{3}$ Prof., Dr., Depto. Fitotecnia e Fitossanidade, FIT/CECA/UFAL. isl@ fapeal.br.

${ }^{4}$ Pesquisadora, Dra., QUI/CCEN/UFAL.

${ }_{5}^{5}$ Prof., Dr., Depto. QUI/CCEN/UFAL. aegs@qui.ufal.br
} 


\section{MATERIALEMÉTODOS}

$\mathrm{O}( \pm)$-rincoforol foi sintetizado no departamento de Química da Universidade Federal de Alagoas (Navarro et al., 2002), utilizando 1bromo-2-metilpropano (MERK), tetrahidrofurano (VETEC) e crotonaldeído (Aldrich). O hexanoato de etila, hexanoato de metila, acetato de pentila e o octanoato de metila foram sintetizados em laboratório a partir dos álcoois e ácidos carboxílicos correspondentes (Aldrich). $\mathrm{O}$ decanoato de metila e o heptanoato de etila foram adquiridos comercialmente (Aldrich).

Frutos maduros de abacaxi das variedades Pérola e Jupi foram aerados por 48 horas, utilizando-se de um dessecador de vidro com capacidade para 7,5 litros ( $25 \mathrm{~cm}$ de diâmetro de boca x $32,5 \mathrm{~cm}$ de altura), o qual permitia a passagem do ar atmosférico, previamente filtrado, na câmara onde estava acondicionado o fruto. Os voláteis liberados pelos frutos foram arrastados pelo vácuo, proporcionado por uma trompa d'água e aprisionados em um tubo ( $14 \mathrm{~cm}$ de comprimento $\mathrm{x} 0,8 \mathrm{~cm}$ de diâmetro) contendo um adsorvente (carvão ativo ou tenax). Os constituintes voláteis foram dessorvidos do adsorvente pela passagem de hexano, e o extrato obtido foi concentrado com nitrogênio seco. Os extratos foram analisados por cromatografia gasosa (HP5890, série II) acoplada à espectrometria de massas HP5970 controlado por um computador ChemStation 50070C. Os componentes químicos presentes nos extratos foram separados em coluna capilar com fase estacionária apolar (polidimetilsiloxano, $15 \mathrm{~m}$ x $0.32 \mathrm{~mm}$ D. I. x $0.5 \mu \mathrm{m}$ espessura do filme de fase estacionária). As zonas de aquecimento do cromatógrafo foram programadas para operar nas seguintes temperaturas: injetor $150{ }^{\circ} \mathrm{C}$, detector (espectrômetro de massas) $270{ }^{\circ} \mathrm{C}$ e forno do cromatógrafo 30 ${ }^{\circ} \mathrm{C}$ por 2 minutos, com velocidade de aquecimento de $8{ }^{\circ} \mathrm{C} \mathrm{min}^{-1}$ até atingir a temperatura de $150^{\circ} \mathrm{C}$. Hélio $\left(1 \mathrm{~mL} / \mathrm{min}^{-1}\right)$ foi utilizado como gás de arraste. A energia de ionização foi de $70 \mathrm{eV}$, e o espectrômetro de massas operou sob o modo "scan". Os compostos voláteis liberados pelos frutos foram identificados por comparação dos tempos de retenção, e espectros de massas de padrões autênticos e/ou espectros, registrados na literatura.

Dois experimentos seqüenciados foram instalados no campo com a finalidade de observar-se o efeito sinérgico do rincoforol associado a seis componentes voláteis do abacaxi. O delineamento estatístico utilizado foi o quadrado latino com oito tratamentos. Para a realização dos experimentos, foram utilizadas armadilhas previamente testadas, de modo a permitir o acesso, mas não a saída, dos besouros do seu interior. As armadilhas foram confeccionadas, modificando-se um balde de plástico com capacidade para 5 litros. Um funil de plástico foi adaptado na tampa, que, por ser provida de rosca, facilitava o trabalho de inspeção. As armadilhas foram instaladas em diferentes localidades ao longo do litoral norte do Estado de Alagoas, sendo enterradas no solo para facilitar o manuseio e diminuir a possibilidade de serem danificadas. A distância média entre armadilhas foi de $5 \mathrm{~km}$. As armadilhas foram inspecionadas a cada 8 dias, contando-se o número de machos e fêmeas capturados em cada uma delas. Após cada inspeção, os pedaços de cana-de-açúcar eram substituídos e as armadilhas trocadas de local, para observar-se o efeito do local e época na captura dos besouros. O primeiro experimento foi realizado no período de 64 dias. O feromônio e os componentes voláteis do abacaxi foram colocados em cápsulas de plástico do tipo "Eppendorf safe-lock®", contendo um furo de $1 \mathrm{~mm}$ na tampa. Em cada cápsula, adicionaram-se $700 \mu \mathrm{L}$ de rincoforol ou $400 \mu \mathrm{L}$ de cada um dos ésteres em estudo. Os tratamentos foram os seguintes:

1) 3 pedaços de abacaxi

2) 1 cápsula com rincoforol

3) 1 cápsula com rincoforol e 3 pedaços de cana-de-açúcar (testemunha)

4) 1 cápsula com rincoforol e 3 pedaços de abacaxi

5) 1 cápsula com rincoforol e 1 cápsula com éster $A$ do abacaxi

6) 1 cápsula com rincoforol e 1 cápsula com éster $B$ do abacaxi

7) 1 cápsula com rincoforol e 1 cápsula com éster $\mathrm{C}$ do abacaxi

8) 1 cápsula com rincoforol e 1 cápsula com éster $\mathrm{D}$ do abacaxi
Onde: éster $\mathrm{A}=$ hexanoato de etila; $\mathrm{B}=$ hexanoato de metila; $\mathrm{C}=$ acetato de pentila, e $\mathrm{D}=$ octanoato de metila.

O segundo experimento foi realizado no período de 64 dias. $\mathrm{O}$ feromônio e os componentes voláteis do abacaxi foram colocados em cápsulas de plástico como descritas no experimento anterior. Em cada cápsula, adicionaram-se $700 \mu \mathrm{L}$ de rincoforol ou $160 \mu \mathrm{L}$ de cada um dos ésteres em estudo. Os tratamentos foram os seguintes:

1) 1 cápsula com rincoforol e 3 pedaços de cana-de-açúcar (testemunha)

2) 1 cápsula com rincoforol e 1 cápsula com ésteres $A+B+C+D+E$

3) 1 cápsula com rincoforol e 1 cápsula com ésteres $A+B+C$

4) 1 cápsula com rincoforol e 1 cápsula com ésteres $A+B+D$

5) 1 cápsula com rincoforol e 1 cápsula com ésteres $A+B+E$

6) 1 cápsula com rincoforol e 1 cápsula com ésteres $C+D+E$

7) 1 cápsula com rincoforol e 1 cápsula com ésteres $A+B$

8) 1 cápsula com rincoforol e 1 cápsula com ésteres D + E

Onde: éster $\mathrm{A}=$ hexanoato de etila; $\mathrm{B}=$ hexanoato de metila; $\mathrm{C}=$ octanoato de metila; $\mathrm{D}=$ decanoato de metila, e $\mathrm{E}=$ heptanoato de metila.

\section{RESULTADOSE DISCUSSÃO}

Flath \& Forrey (1970), utilizando a técnica de extração líquido-líquido, identificaram quarenta e cinco compostos em frutos da variedade Smooth Cayenne, sendo, na maioria, compostos oxigenados. Dos dezoito compostos aqui relacionados (Tabela 1), apenas seis foram também identificados por Flath \& Forrey (1970): hexanoato de metila, hexanoato de etila, heptanoato de etila, 3metiltiopropionato de etila, octanoato de metila e octanoato de etila. A técnica de aeração com fase sólida de carvão ativo ou tenax para adsorção dos voláteis de pedaços de abacaxi proporcionou a obtenção de compostos de função éster, confirmando, segundo Rochat et al.(1991), serem voláteis provenientes da fermentação alcoólica do material vegetal.

TABELA 1. Componentes voláteis identificados em frutos de duas variedades de abacaxi (Pérola e Jupi). Maceió, 2002.

\begin{tabular}{|c|c|c|}
\hline Compostos & $\begin{array}{c}\% \text { do composto no } \\
\text { extrato (abacaxi } \\
\text { em pedaços) }\end{array}$ & $\begin{array}{c}\% \text { do composto } \\
\text { no extrato } \\
\text { (abacaxi } \\
\text { inteiro) }\end{array}$ \\
\hline 2-metilbutanoato de etila & 25,04 & - \\
\hline acetato de 2-metilbutila & 9,79 & - \\
\hline hexanoato de metila & 17,17 & 41,77 \\
\hline hexanoato de etila & 16,95 & 15,12 \\
\hline 2-nonanona & 0,80 & - \\
\hline heptanoato de etila & 0,45 & - \\
\hline 3-metiltiopropionato de etila & 0,90 & - \\
\hline octenoato de metila & 0,45 & 0,43 \\
\hline octanoato de metila & 8,95 & 7,18 \\
\hline octenoato de etila & 1,25 & - \\
\hline octanoato de etila & 9,15 & 27,67 \\
\hline composto desconhecido 1 & 4,13 & - \\
\hline decanoato de metila & 1,18 & - \\
\hline composto desconhecido 2 & 3,75 & - \\
\hline decanoato de etila & 0,17 & - \\
\hline pentanoato de metila & - & 4,27 \\
\hline heptanoato de metila & - & 3,14 \\
\hline hexenoato de etila & 0,33 & - \\
\hline $\begin{array}{c}\text { Média da quantidade total dos } \\
\text { componentes na amostra }\end{array}$ & $\begin{array}{c}787,44 \\
\text { ng/amostra }\end{array}$ & $\begin{array}{c}359,81 \\
\text { ng/amostra }\end{array}$ \\
\hline
\end{tabular}

${ }^{1}$ Quantidade em $1 \mu \mathrm{L}$ de extrato injetado no cromatógrafo gasoso. 
Os componentes encontrados em maiores proporções, obtidos da aeração do abacaxi em pedaços, foram 2-metil butanoato de etila, hexanoato de metila, hexanoato de etila, octanoato de metila e octanoato de etila. Por outro lado, hexanoato de metila, hexanoato de etila e octanoato de etila foram os mais abundantes obtidos por aeração do abacaxi inteiro. Esses resultados indicam que, exceto pela presença de 2metil butanoato de metila, os dois extratos têm os mesmos componentes em maior abundância (Tabela 1). Verificou-se também que a exposição da polpa do fruto na aeração do abacaxi em pedaços proporcionou maior número de voláteis identificados do que os obtidos pela aeração do abacaxi inteiro.

Para estudar o efeito sinérgico do rincoforol e os constituintes voláteis do abacaxi na captura de adultos de $R$. palmarum, utilizaram-se os ésteres em maior abundância nos dois extratos. Hexanoato de metila, hexanoato de etila, acetato de pentila e octanoato de metila foram sintetizados no Departamento de Química da Universidade Federal de Alagoas, enquanto heptanoato de etila e decanoato de metila foram adquiridos comercialmente.

A análise dos resultados do primeiro experimento instalado no campo mostrou que não houve efeito significativo do local e da época na captura de $R$. palmarum. Os índices de captura dos tratamentos rincoforol e toletes de cana-de-açúcar, e rincoforol e pedaços de abacaxi mostraram não possuir diferenças significativas entre si, contudo apresentaram índices de captura mais eficientes que os demais (teste de Tukey, $\mathrm{P}<0,05)$ (Figura 1).

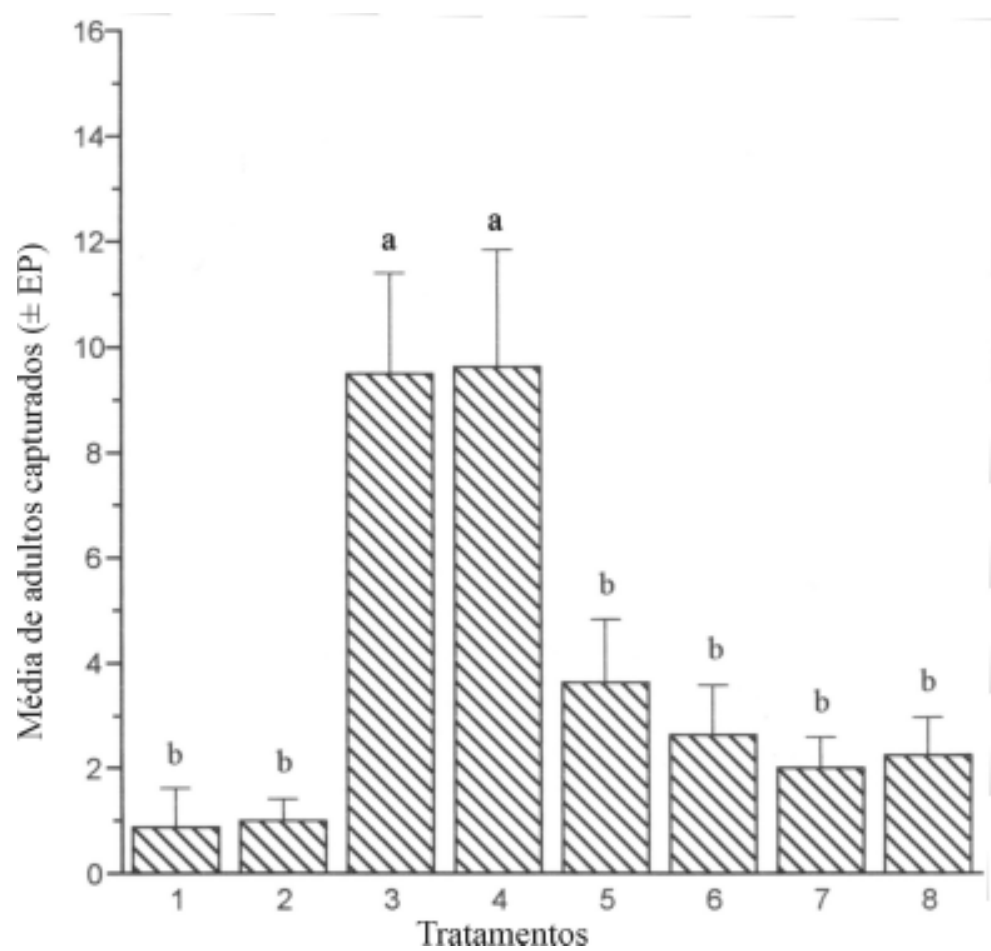

FIGURA 1. Média de adultos de Rhynchophorus palmarum capturados em armadilhas iscadas com rincoforol associado a vários ésteres do abacaxi, ao fruto de abacaxi e ao colmo de canade-açúcar. $1=$ abacaxi; 2 = rincoforol; $3=$ rincoforol + canade-açúcar (testemunha); $4=$ rincoforol + abacaxi; $5=$ rincoforol + éster A; $6=$ rincoforol + éster $\mathrm{B} ; 7=$ rincoforol + éster $\mathrm{C} ; 8=$ rincoforol + éster $\mathrm{D}$. Médias seguidas da mesma letra não diferem entre si, pelo teste Tukey $(\mathrm{P}<0,05)$. Maceió, 2002.

No segundo experimento, também não houve efeito significativo do local e da época na captura de $R$. palmarum. As armadilhas iscadas com rincoforol e toletes de cana-de-açúcar apresentaram índices de captura significativamente superiores aos demais tratamentos, os quais não diferiram entre si (teste de Tukey, $\mathrm{P}<0,05$ ) (Figura 2).

Os resultados deste estudo indicaram que o rincoforol, combinado com pedaços de cana-de-açúcar ou pedaços de abacaxi, tornaram as armadilhas mais eficientes, apresentando índices de captura superiores aos obtidos pelas armadilhas iscadas apenas com rincoforol ou apenas com pedaços de cana-de-açúcar. Sugere-se que os componentes voláteis, oriundos da fermentação da cana-de-açúcar ou do abacaxi, quando combinados com o rincoforol, são os responsáveis pela atração dos adultos de R. palmarum para as armadilhas. Estes resultados coincidem com os de vários autores, os quais foram unânimes em afirmar que o feromônio sozinho não foi eficiente na captura de adultos de $R$. palmarum, mesmo quando se liberou no ambiente cerca de $30 \mathrm{mg} / \mathrm{dia}$ (Oehlschlager et al., 1992, 1993; Gries et al., 1993; Jaffé et al., 1993; Perez et al., 1994; Oehlschlager et al., 1995). No entanto, esses mesmos autores afirmaram que quando o feromônio foi utilizado juntamente com pedaços de tecidos da planta e acetato, houve um aumento significativo no número de insetos capturados, sugerindo que existe uma interação entre os componentes químicos da planta e o feromônio produzido pelo inseto, aumentando a atratividade das armadilhas em até 20 vezes.

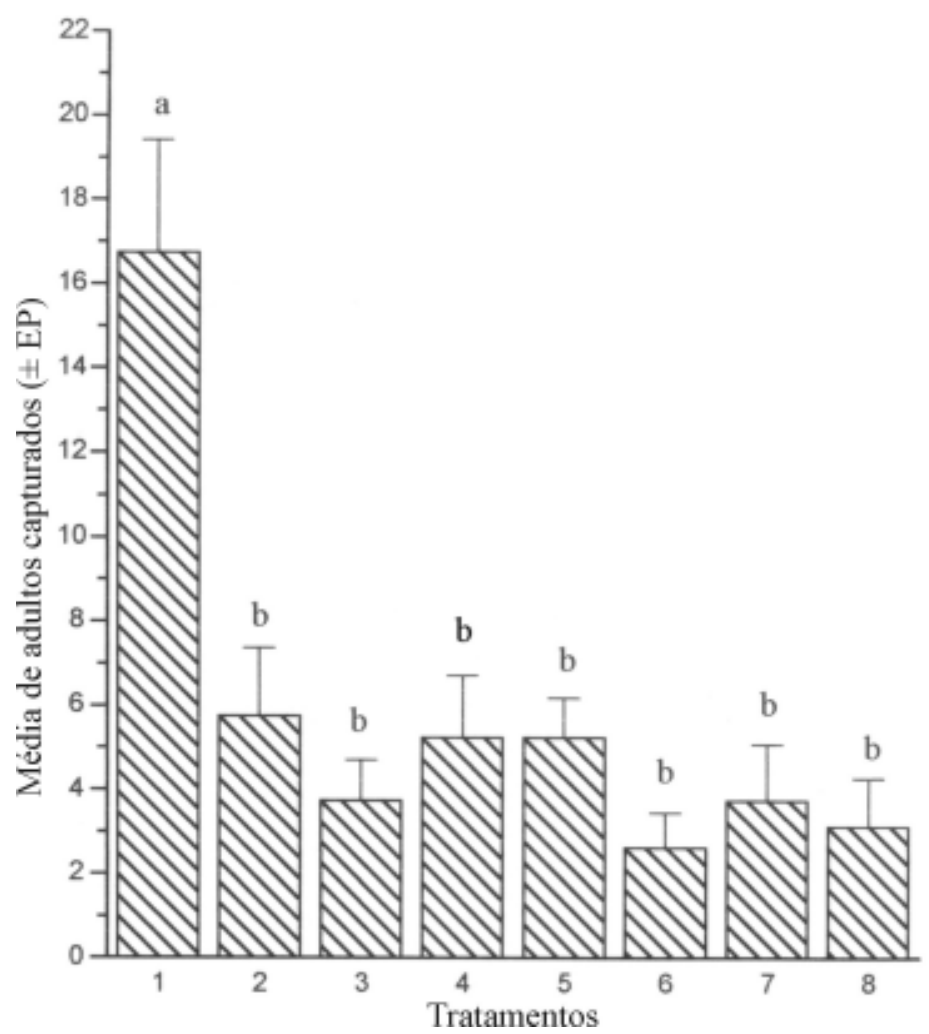

FIGURA 2. Média de adultos de Rhynchophorus palmarum capturados em armadilhas iscadas com rincoforol associado a cana-deaçúcar e vários ésteres do abacaxi. $1=$ rincoforol + cana-deaçúcar (testemunha); 2 = rincoforol + ésteres $\mathrm{A}+\mathrm{B}+\mathrm{C}+\mathrm{D}+\mathrm{E}$; $3=$ rincoforol + ésteres $\mathrm{A}+\mathrm{B}+\mathrm{C} ; 4=$ rincoforol + ésteres $\mathrm{A}+\mathrm{B}+\mathrm{D} ; 5=$ rincoforol + ésteres $\mathrm{A}+\mathrm{B}+\mathrm{E} ; 6=$ rincoforol + ésteres $\mathrm{C}+\mathrm{D}+\mathrm{E} ; 7=$ rincoforol + ésteres $\mathrm{A}+\mathrm{B} ; 8=$ rincoforol + ésteres D+E. Médias seguidas da mesma letra não diferem entre si, pelo teste Tukey $(\mathrm{P}<0,05)$. Maceió, 2002.

Jaffé et al. (1993) relataram que, em laboratório, a mistura de $68 \%$ de etanol, $27 \%$ de acetato de etila e $5 \%$ de pentano atraiu os adultos de $R$. palmarum tanto quanto os tecidos de plantas de coqueiro. Entretanto, quando testados em condições de campo, nenhum deles, separados ou em combinação, foram atraentes, sugerindo que estes compostos atraem os besouros apenas a curta distância da fonte de odor. Dessa forma, o rincoforol, associado a outros odores é responsável pela atração e orientação do inseto a longa distância. Os resultados aqui obtidos mostraram que não houve diferenças significativas quanto ao número de machos e de fêmeas capturados nas armadilhas, coincidindo com os de Tiglia et al. (1998), Rochat et al. (1991), Oelhschlager et al. (1993) e Jaffé et al. (1993), os quais afirmaram que o feromônio de agregação atrai igualmente ambos os sexos. 


\section{CONCLUSÕES}

1) $\mathrm{O}( \pm)$-rincoforol, combinado com pedaços de abacaxi, torna as armadilhas tão atraentes quanto aquelas contendo ( \pm )-rincoforol com pedaços de cana-de-açúcar.

2) Não houve aumento significativo na captura dos insetos pelo uso do ( \pm )-rincoforol associado aos voláteis mais abundantes do extrato hexânico obtido da aeração do abacaxi, se comparada à captura dos insetos nas armadilhas iscadas com ( \pm )-rincoforol associada à canade-açúcar ou abacaxi.

\section{AGRADECIMENTOS}

Os autores agradecem à Fundação de Amparo à Pesquisa do Estado de Alagoas (FAPEAL), ao CNPq e a CAPES. Agradecem também ao professor Geraldo Veríssimo Barbosa, pela indicação dos delineamentos estatísticos utilizados nos experimentos de campo.

\section{REFERÊNCIASBIBLIOGRÁFICAS}

FLATH, R.A; FORREY, R.R. Volatile components of smooth cayenne pineapple. Journal of Agricultural and Food Chemistry, Washington, v.18, p.306-309, 1970.

GIBLIN-DAVIS, R.M.; GERBER K.; GRIFFITH, R. Laboratory rearing of Rhynchophorus cruentatus and $R$. palmarum (Coleoptera: Curculionidae). Florida Entomologist, Winter Haven, v.72, p.480-489, 1989.

GIBLIN-DAVIS, R.M.; WEISSLING, T.J.; OEHLSCHLAGER, A.C.; GONZALEZ, L.M. Field response of Rhynchophorus cruetatus (Coleoptera: Curculionidae) to its aggregation pheromone and fermenting plant volatiles. Florida Entomologist, Winter Haven, v.77, p.164$177,1994$.

GRIES, G; GRIES, R.; PEREZ,A.L.; OEHLSCHLAGER,A.C.; GONZALES, L.M.; PIERCE JR., H.D.; KOUDA, M.; ZEBEYOU, M.; NANOU, N. Agregation pheromone of the African palm weevil, Rhynchophorus phoenicis F. Naturwissenschafte, New York, v.80, p.90-91, 1993.

GRIES, G; GRIES, R.; PEREZ,A.L.; GONZALES, L.M.; PIERCE JR., H.D.; OEHLSCHLAGER, A.C.; RHAINDS, M.;ZEBEYOU, M.; KOUAME, B. Ethyl propionate: synergic kairomone for African palm weevil, Rhynchophorus phoenicis L. (Coleoptera: Curculionidae). Journal of Chemical Ecology, New York, v.20, p.889-897, 1994.

GRIFFITH, R. The relationship between the red ring nematode and the palm weevil. Journal of the Agricultural Society of Trinidad and Tobago, Port-of-Spain, v.68, p.342-356, 1968.

GUNATILAKE, R.; GUNAWARDENA, N.E. Ethyl alcohol: a major attractant of red weevil Rhynchophorus ferrugineus. Proceedings of the Sri Lanka Association on Advanced Science, 42nd Ann. Sess. p.70, 1986.

HAGLEY, E.A.C. On the life history and habitats of the palm weevil Rhynchophorus palmarum (L.). Annals of the Entomological Society of America, v.58, p.22-28, 1965.

JAFFÉ, K.; SÁNCHEZ, P.; CERDA,H.; HERNÁNDEZ, J.V.; JAFFÉ, R.; URDANETA, N.; GUERRA, G.; MARTÍNEZ, R.; MIRAS, B. Chemi- cal ecology of the palm weevil Rhynchophorus palmarum (L.) (Coleoptera: Curculionidae): attraction to host plants and to a maleproduced aggregation pheromone. Journal of Chemical Ecology, New York, v.19, p.1703-1720, 1993.

NAVARRO, D.M.A.F.; MURTA, M.M.; DUARTE, A.G.; LIMA, I.S.; NASCIMENTO, R.R.; SANT'ANA, A.E.G. Aspectos práticos relacionados ao uso do rincoforol, o feromônio de agregação da broca-doolho-do-coqueiro Rhynchophorus palmarum L. (Coleóptera: Curculionidae) no controle de pragas do coqueiro. Análise de sua eficiência no campo. Química Nova, São Paulo, v.25, n.1, p.32-36, 2002.

OEHLSCHLAGER, A.C.; PIERCE, H.D.; MORGAN, B.; WIMALARATNE, P.D.C.; SLESSOR, K.N.; KING, G.G.S.; GRIES, R.; BORDEN, J.H.; JIRON, L.F.; CHINCHILA, C.M.; MEXZON, R.G. Chirality and field activity of Rhynchophorol, the aggregation pheromone of the American palm weevil. Naturwissenschaften, New York, v.79, p.134-135, 1992.

OEHLSCHLAGER,A.C.; CHINCHILLA, C.M.; GONZÁLEZ,L.M.; JIRON, L.F.; MEXZON, R.; MORGAN, B. Development of a pheromonebased trapping system for Rhynchophorus palmarum (L.) (Coleoptera: Curculionidae). Journal of Economic Entomology. Maryland, v.86, p.1381-1392, 1993.

OEHLSCHLAGER, A.C.; MCDONALD, R.S.; CHINCHILLA, C.M.; PATSCHKE, S.N. Influence of a pheromone-based mass-trapping system on the distribution of Rhinchophorus palmarum (Coleoptera:Curculionidae) in oil palm. Environmental Entomolog,. Lanham, v.24, p.1005-1012, 1995.

PEREZ,A.L.; GRIES, G; GLIBLIN-DAVIS, R.M.; OEHLSCHLAGER,A.C. Pheromone chirality of the African palm weevil, Rhynchophorus phoenicis (F.) and the palmetto weevil, Rhynchophorus cruentatus (F.) (Coleoptera: Curculionidae). Journal of Chemical Ecology, New York, v.20, p.2653-2671, 1994

PEREZ, A.L.; HALLETT, R.H.; GRIES, R.; GRIES, G.; OEHLSCHLAGER, A.C.; BORDEN, J.H. Pheromone chirality of Asian palm weevils, Rhynchophorus ferrugineus (OLIV.) and $R$. vulneratus (PANZ.) (Coleoptera:Curculionidae). Journal of Chemical Ecology, New York, v.22, p.357-368, 1995.

ROCHAT, D.; GONZÁLEZ, V.A.; MARIAU, D.; VILLANUEVA, G.A.; ZAGATTI, P. Evidence for male-produced aggregation pheromone in American palm weevil, Rhynchophorus palmarum (L.) (Coleoptera: Curculionidae). Journal of Chemical Ecology, New York, v.17, p.12211230, 1991.

SÁNCHEZ, P.A.; CERDA, H. El complejo Rhynchophorus palmarum (L.) (Coleoptera: Curculionidae) - Bursaphelenchus cocophilus (Cobb) (Tylenchida: Aphelenchoididae), en palmeras. Boletin de Entomologia Venezuelana, v.8, p.1-18, 1993.

TIGLIA, E.A.; VILELA, E.F.; MOURA, J.I.L.; ANJOS, N. Eficiência de armadilhas com feromônio de agregação e cana-de-açúcar na captura de Rhynchophorus palmarum (L). Anais da Sociedade Entomológica do Brasil, Londrina, v.27, p.177-183, 1998.

WATTANAPONGSIRI, A. A revision of the genera Rhynchophorus and Dynamis (Coleoptera:Curculionidae). Department of Agricultural Science Bulletin, Bangkok, v.1, p.1-328, 1966. 